\title{
Electrochemical and corrosion behavior of two chromium dental alloys in artificial bioenvironments
}

\author{
Alexandra Banu ${ }^{1, *}$, Cristina Juganaru ${ }^{1}$, and Maria Marcu $^{2}$ \\ ${ }^{1}$ UPB, Manufacturing Engineering Department, 313 Splaiul Independentei, Romania \\ 2"Ilie Murgulescu" Institute of Physical Chemistry of the Romanian Academy, 202 Splaiul \\ Independentei, Romania
}

\begin{abstract}
The purpose of this study is to compare the corrosion and tarnish behavior of NiCrMo and CoCrMo cast dental alloys in artificial bio environments. The cobalt chromium alloys are known and used in dentistry for many years, but its difficult machinability because of the strength and hardness, is an argument for scientists to study alternative materials with comparable biocompatibility. On the other hand, for dentistry devices beside corrosion behavior is important the aesthetic so, the used alloys have to preserve their shining and do not stain. The corrosion resistance has been evaluated using the Atomic mass spectroscopy method for ion release determination, the anodic polarization curves and the open circuit potential - time monitoring for corrosion behavior evaluation and optical microscopy for the structure analysis. The tarnish tendency of alloys was estimated using the method of cyclic immersion with frequency of 10 seconds for each minute during 72 hours in $\mathrm{Na}_{2} \mathrm{~S}$ containing solution. The most important conclusion is that the alloys are comparable from corrosion and tarnish point of view, but we recommend to use the nickel base alloy only for orthodontic devices implanted for short periods of time, because of higher quantity of released ions.
\end{abstract}

\section{Introduction}

Mechanical properties of alloys used in dental crown and bridges applications differ from those of porcelain devices [1-3]. The first materials have to be tough and rigid and the second one, on the contrary, because these properties make difficult the finishing steps as grinding and polishing. Dental alloys must have excellent corrosion resistance to the oral environment, which is highly important for maintaining biocompatibility throughout the time of use of the device. From this point of view, is not so important and dangerous the ions generation because of corrosion, how is their toxicity, their poisoning power for human body $[4,5]$. Negative effect of dental alloys in the oral cavity is their capability to produce local or systematic changes that may be short term or long term or systematic. Interactions between the oral environment and dental alloys can also generate some discomforts like metallic taste,

\footnotetext{
*Corresponding author: alexandrabanu14@yahoo.com
} 
discoloration of teeth, galvanic currents, oral lesions, dermatitis hypersensitivity, dental implant rejection, and generating tumors and decay [5-6].

Because the noble metals (gold, palladium, silver) became less and less accessible for many people, in the recent decades, new alternative and cheaper materials came to dental market. The materials used in oral environment must be completely tarnish-resistant, they must be chemically and electrochemically inert in contact with a very big variety of foods, that means a large $\mathrm{pH}$ domain $[7,8]$. While corrosion behavior of a material is generally understood, the tarnishing is a less used notion. Tarnish can be defined as a surface discoloration, in fact color changes, or decrease in luster of a dental alloy because of the formation of a surface product.

The main task of this work is to evaluate how two nickel and cobalt chromium alloys behave in oral cavity from metallic ions release and tarnish point of view.

\section{Experimental details}

The experimental samples have been cut from two chromium molybdenum cast dental alloys with the chemical composition shown in Table 1.

Table 1. Chemical composition (\% weight) of studied alloys.

\begin{tabular}{|c|c|c|c|c|c|c|c|c|c|c|}
\hline $\begin{array}{c}\text { Sample } \\
\text { label }\end{array}$ & Alloy & $\begin{array}{c}\mathrm{Cr} \\
\%\end{array}$ & $\begin{array}{c}\mathrm{Mo} \\
\%\end{array}$ & $\begin{array}{c}\mathrm{Fe} \\
\%\end{array}$ & $\begin{array}{c}\mathrm{Si} \\
\%\end{array}$ & $\begin{array}{c}\mathrm{Mn} \\
\%\end{array}$ & $\begin{array}{c}\mathrm{Al} \\
\%\end{array}$ & $\begin{array}{c}\mathrm{Ti} \\
\%\end{array}$ & $\begin{array}{c}\mathrm{Co} \\
\%\end{array}$ & $\begin{array}{c}\mathrm{Ni} \\
\%\end{array}$ \\
\hline 1. & $\mathrm{NiCr}$ & 19.2 & 5.2 & 2 & 2.09 & 1.5 & 0.6 & 0.34 & 0.4 & base \\
\hline 2. & $\mathrm{CoCr}$ & 28.1 & 4.2 & - & 1.01 & 0.6 & - & 0.45 & base & - \\
\hline
\end{tabular}

The CoCrMo alloy (sample 2) is close to a known dental material, Stellite 21, but with less Molybdenum content ( $4 \%$ of Molybdenum instead of $6 \%$ ).

Before each test, the samples were mechanically prepared according with the practical instruction from dentistry laboratories.

\subsection{Techniques, solutions, samples}

For structural characterization of materials was used optical microscopy after electrochemical etching of surfaces using $2 \%$ chromic acid solution. Have been used the following tests for characterization of corrosion behavior of studied alloys:

1. The Stationary immersion test during $168 \mathrm{~h}$ at $(37 \pm 1)^{\circ} \mathrm{C}$ was used in order to evaluate the corrosion rates and pitting corrosion resistance [2]. For corrosion rate have been used two ways: mass loss and the electrolyte's analysis after immersion time. As corroding solution was used, according to ISO 10271:2011, lactic acid $\mathrm{C}_{3} \mathrm{H}_{6} \mathrm{O}_{3}(0.1 \mathrm{M})$; Natrium chloride, $\mathrm{NaCl}(0.1 \mathrm{M}) ; \mathrm{H}_{2} \mathrm{O}$ grade 2, according to ISO 3696. All reagents were pro analysis grade of quality. The samples were cut at $32 \times 10 \times 1.5 \mathrm{~mm}$ of dimension. Corrosion rates were done by weighing using the analytical balance $\left(10^{-4} \%\right.$ accuracy $)$ before and after immersion and by solution analysis using the Atomic mass spectroscopy method for ion release determination.

2. Time monitoring of open circuit potential (OCP) during $168 \mathrm{~h}$ and the cyclic potentiodynamic polarization method were done in artificial saliva with chemical composition according [2] ( $\mathrm{NaCl}, \mathrm{KCl}, \mathrm{CaCl}_{2} \times 2 \mathrm{H}_{2} \mathrm{O}, \mathrm{NaH}_{2} \mathrm{PO}_{4} \mathrm{H}_{2} \mathrm{O}, \mathrm{Na}_{2} \mathrm{Sx}_{9} \mathrm{H}_{2} \mathrm{O}$, Urea; $\mathrm{H}_{2} \mathrm{O}$ grade 2); all reagents were pro analysis grade of quality; as reference electrode was used the saturated calomel electrode (sce). Were used cylindrical samples with a total surface area of $1 \mathrm{~cm}^{2}$; the electrical contact was made by screw (a threaded hole was made at one end of the 
sample). Potentiodynamic polarization test was performed using a Radiometer potentiostat/ galvanostat PGP 201 and acquired data were processed with Voltalab specialized software. The potential was scanned between $-250 \mathrm{mV}$ and $+1000 \mathrm{mV}$ forward and back with a scan rate of $0.6 \mathrm{~V} / \mathrm{h}$.

3. Sulfide tarnish test (cyclic immersion) [2], was performed using cylindrical samples $(\mathrm{L}=0.5 \mathrm{~mm} ; \Phi=10 \mathrm{~mm}$ ) with immersion frequency of 10 seconds for each minute, during 72 hours at room temperature. As a staining solution was used $\mathrm{Na}_{2} \mathrm{~S} \times 7 \mathrm{H}_{2} \mathrm{O}$ and distilled water grade 2. The device for cyclic immersion was built in our laboratory. A vertical wheel is driven by a step-by-step electric motor so that the specimen is immersed in the test solution 10 seconds for each minute (a cycle consists in 10 seconds of immersion and 50 seconds of air exposure).

\section{Results and Discussions}

\subsection{Structure}

As is known, the chemical composition of an alloy is not determining factor for the physical properties of the material [9] and from this point of view very important is the material's structure. In such alloys, the cobalt element affects the modulus, strength and hardness of the alloy, the carbon increases the hardness because a lot of elements such as chromium, molybdenum and cobalt react with carbon to form carbides, affecting the properties of alloys. In addition, the silicon and manganese increase the liquidity and stability and the molybdenum and chromium are benefic for corrosion resistance in the chloride ions presence [10]. As shown in the Fig. 1, both alloys present dendritic structure with an important carbides quantity. The nickel base alloy (Fig. 1a) seems to have a bigger quantity of carbides compared with the cobalt alloy and the structure shows fine elements, oriented in the solidification direction. The cobalt alloy (Fig. 1b) has an austenitic matrix composed of the solid solution of cobalt and chromium, and the dendritic structure formed by different chemical segregations. Despite the fact that it is the most studied non precious dental alloy, yet many works are devoted to the study of its structure [11-13]. Most of segregated phases are carbides with different formal oxidation number, compounds from $\mathrm{MC}$ to $\mathrm{M}_{23} \mathrm{C}_{6}$ with specific morphologies such as spherical with discontinuous arrangement or agglomerated within grain boundaries.
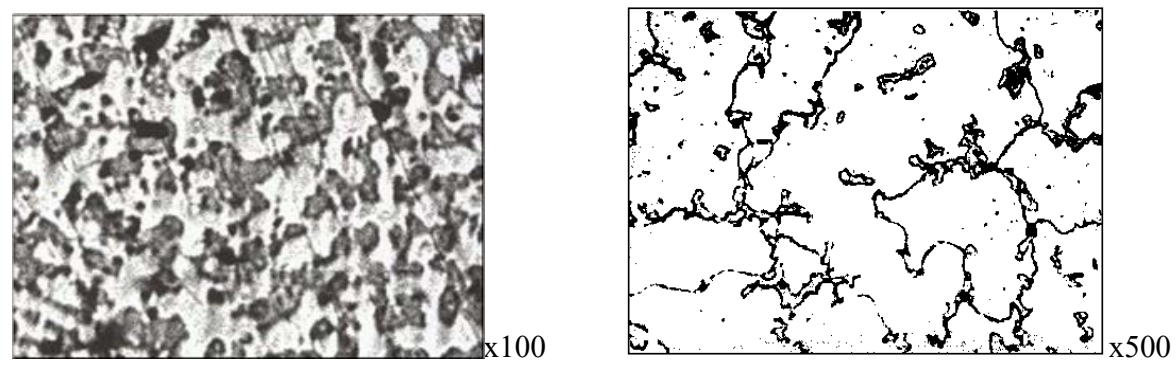

Fig. 1. Microstructure of samples: (a) NiCrMo alloy and (b) CoCrMo alloy, after electrochemical etching using $2 \%$ of chromium acid solution.

\subsection{Stationary immersion test}

The Stationary immersion test consists, according to ISO 10271, in determination of mass loss of the samples by weighing before and after immersion, and in determination by analyze of electrolyte of metallic ions released. After $168 \mathrm{~h}$ of immersion in synthetic bio fluid at 
$(37 \pm 1)^{\circ} \mathrm{C}$, the quantities of metallic ions released, expressed as $\mu \mathrm{g} \mathrm{cm}^{-2}$ of immersed surface are presented in Table 2. As is shown in the table, the nickel alloy released an important quantity of ions in $168 \mathrm{~h}$ of immersion (about $93 \mu \mathrm{g} \mathrm{cm}$ ). Despite of the fact that the nickel has an important toxicity potential, some studies $[4,14,15]$ reveal that nickel is a moderate allergen and no evidence of toxicity associated with the intraoral use of nickel-containing dental alloys is found. It is concluded that, subject to use it according to established techniques, the dental alloys with nickel do not pose a risk to patients or members of the dental team. On the other hand, the quantity of nickel ions ingested from food or water is higher than that from oral devices or from dental reconstruction [4].

Table 2. The ions release, expressed in $\mu \mathrm{g} \mathrm{cm}^{-2}$, after $168 \mathrm{~h}$ of immersion in lactic acid solution at $(37 \pm 1){ }^{\circ} \mathrm{C}$ - Atomic mass spectroscopy.

\begin{tabular}{|l|c|c|c|c|}
\hline \multirow{2}{*}{ Alloy } & \multicolumn{4}{|c|}{ Release, $\mu \mathrm{g} \mathrm{cm}^{-2}$} \\
\cline { 2 - 5 } & $\mathrm{Ni}$ & $\mathrm{Co}$ & $\mathrm{Cr}$ & $\mathrm{Mo}$ \\
\hline NiCrMo & 77.85 & - & 4.234 & 4.4 \\
\hline CoCrMo & - & 0.314 & 0 & 0 \\
\hline
\end{tabular}

Compared with nickel alloy, the cobalt alloy loses a very small quantity of metallic ions (less than $1 \mu \mathrm{gcm}^{-2}$ in $168 \mathrm{~h}$ ) and this shows a better behavior as biocompatible material for cobalt chromium alloy. Table 3 presents the corrosion rates $\left(\mathrm{gm}^{-2} \mathrm{~h}^{-1}\right)$ determined by weight loss of the samples. This values, are in good agreement with those obtained by Okazaki and Gotoh [16] in similar conditions.

Table 3. Corrosion rates after $168 \mathrm{~h}$ of immersion in lactic acid solution.

\begin{tabular}{|c|c|c|c|c|}
\hline Alloy & $\begin{array}{c}\text { Immersed } \\
\text { surface } \mathrm{cm}^{2}\end{array}$ & $\begin{array}{c}\text { Weight loss } \\
\mathrm{mgcm}^{-2}\end{array}$ & $\begin{array}{c}\text { Corrosion rate } \\
\mathrm{gm}^{-2} \mathrm{~h}^{-1}\end{array}$ & $\begin{array}{c}\text { Penetration rate } \\
\mathrm{mmpy}\end{array}$ \\
\hline NiCrMo & 7.8 & 0.395 & 0.0235 & 0.02445 \\
\hline CoCrMo & 7.08 & 0.0876 & 0.00522 & 0.00574 \\
\hline
\end{tabular}

From practical point of view, an important design parameter is the corrosion rate expressed as the rate of decreasing of the oral implant thickness, in millimeters per year (penetration rate). The average values of penetration rates, calculated as arithmetic average of three values, highlighted superior behavior of cobalt alloy. The value of 0.005 millimeters per year (mmpy) falls this alloy in class II, "Very resistant", according to STAS 9684-82, while the nickel alloy presents a corrosion rate of five times higher and it falls in class III, "Resistant". The pitting corrosion evaluation was made by optical inspection at a magnification of 10 times and we found that the cobalt alloy was perfect resistant (no pits), while the nickel alloy is sensible, an incipient corrosion attack was observed.

\subsection{Electrochemical tests}

Have been run two types of electrochemical tests: time evolution of open circuit potential (OCP) for 90 hours and cyclic polarization, toward anodic direction and back, in order to calculate corrosion rates and estimate the sensitivity to pitting corrosion related to passivation - repassivation tendency. Electrochemical tests were performed in artificial saliva environment. Figure 2 shows the time evolution of OCP for both alloys and highlights the following aspects: 
- The instantaneous values of mixed potentials are positives at about $+0,24 \mathrm{~V} /$ sce and $+0,48 \mathrm{~V} / \mathrm{sce}$, respectively, this important difference is given by the higher content of chromium in CoCrMo alloy $(28 \% \mathrm{Cr})$ compared with $18 \% \mathrm{Cr}$ in NiCrMo alloy.

- In the first 20 hours, both alloys presented an activation tendency, OCP moved toward electronegative values which mean that the native passive superficial film is quasi-stable in the presence of chlorine ions. As was shown [16] in aqueous environments, the chromium is subject of so called hydro oxolation reactions which in the absence of chloride ions reinforce the passive film, but when the chloride ions are present, they damage this film. This behavior of damage and reinforce of passive film is highlighted by the oscillation of OCP toward electronegative and electropositive values during immersion period.

- After about 70 hours of immersion the OCP values are approximately stabilized around $0 \mathrm{~V} / \mathrm{sce}$ for NiCrMo alloy and around $+0,150 \mathrm{mV} / \mathrm{sce}$ for CoCrMo alloy, respectively. Correlating this behavior with cyclic anodic polarization test, both alloys tend to passivity.

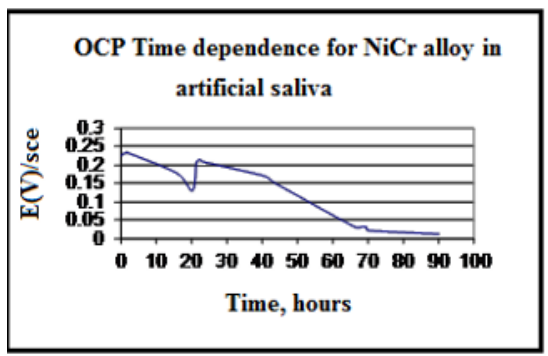

(a)

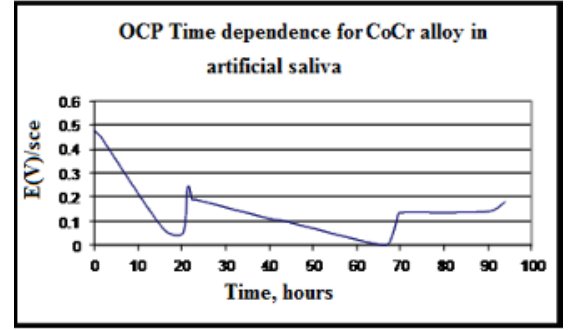

(b)

Fig. 2. Time evolution of the open circuit potential in artificial saliva environment for (a) NiCrMo alloy and (b) CoCrMo alloy .

The Figure 3 shows potentiodynamic polarization curves performed in artificial saliva.

During anodic polarization sequence of the curve, the surface of sample oxidizes and, as can be seen in the figure 3, the corrosion density current does not rises exponentially according Buttler Volmer equation, in fact it is limited diffusion density current with values less than $1 \mathrm{mAcm}^{-2}$. During the reverse scanning of potentials, toward the electronegative values, despite of the reduction processes on the surface, the current density remains at low values, meaning that the surface of sample is still passive. It is the effect of oxygen affinity of chromium and hydro oxolation processes, as was described above and in reference [17]. Despite of chloride ions presence, both alloys are passive in a wide range of potentials, the most important differences between its are the values of difusion limited current density (passive current density).

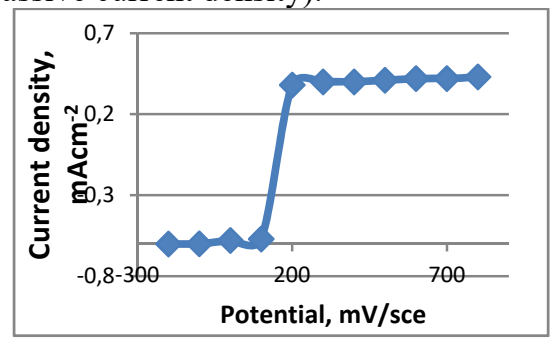

(a)

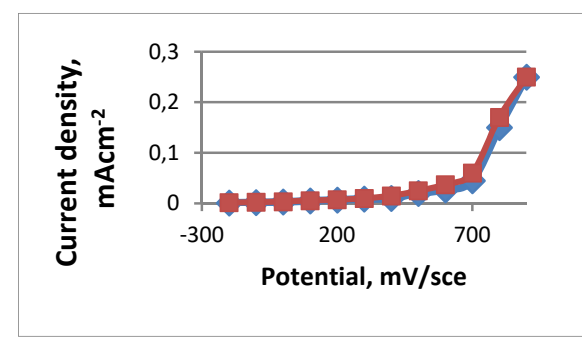

(b)

Fig. 3. Potentiodynamic polarization curves performed in artificial saliva for (a) NiCrMo alloy and (b) CoCrMo alloy .

A higher chromium concentration in the CoCrMo alloy is benefic for an easier passivation, the limited current density is less than $50 \mu \mathrm{Acm}^{-2}$ while for NiCrMo alloy this parameter is about 8 time higher, around of $400 \mu \mathrm{Acm}^{-2}$. Concerning to the corrosion rates 
obtained by Tafel slopes, these values are in agreement with that obtained by weighing of samples, at about 5-6 $\mu \mathrm{m}$ per year for CoCrMo alloy and at $15-20 \mu \mathrm{m}$ per year for NiCrMo alloy.

\subsection{Tarnish test}

Tarnish can be defined as a surface discoloration, or decrease in lustre of a dental alloy because of the corrosion product formation on the surface. Several synthetic saliva solutions, as well as aggressive solutions have been used in attempts to identify in accelerated tests the conditions under which an alloy tarnishes [8]. Since chlorides are present in saliva and certain foods such as fish and eggs have high sulphur content, both chloride and sulphide solutions are commonly chosen as immersion test media. Ranking of alloys in terms of susceptibility to such tarnishing has been recorded subjectively, by comparing colour changes visually. After 72 hours of cyclic immersion in chloride and sulphide containing solution, the surfaces of tested samples are not modified, no color changes or loss of shine. The tested alloys are similar from tarnish resistance point of view.

\section{Conclusions}

Chromium dental alloys used in the oral environment may be affected by electrochemical corrosion processes, so their corrosion behavior can be predicted from results of electrochemical testing. The cobalt chromium alloy revealed an excellent behavior. The nickel chromium alloy tends in time, in artificial saliva, to the electrochemical activity, by movement of OCP toward electronegative potential range, but the corrosion rate is relatively low related to the quantity of released ions. Because of that and correlated with their good tarnish behavior, the nickel chromium alloys can be used for production of dental devices which remain for short time in contact with the oral environment.

\section{References}

1. ASTM F75, Book of Standards Volume: 13.01 F75-12

2. $* * *$ ISO 10271:2011, Dentistry - Corrosion Test Methods For Metallic Materials

3. C. Manaranche, H. Hornberger, Dental Materials 23, 1428-1437 (2007)

4. J. C. Setcos, A.B. Mahani, L. Silvio, et all, Dental Materials 22, 1163-1168 (2006)

5. E. Vasilescu, P. Drob, I. Cinca, et all, Rev. Chim. 61, 168-171 (2010)

6. M. Mikulewicz, K. Chojnacka, Biol Trace Elem Res 142, 865-889 (2011)

7. N. Rin, I. Bau, S. Miko, M. Papi, et all, Coll. Antropol. 27 (2), 99-106 (2003)

8. W. L. Leon, G.W. Stanford, GoldBull. 14 (1), 13-18 (1981)

9. K. Asgar, F. A. Peyton, J. Dental Research 40, 63-72 (1961)

10. J.C. Wataha In Craig RG, JM Powers editor. Restorative dental materials. The 11th edition. (St Louis: Mosby, 163-184, 2002)

11. J. Marciniak, Marciniak J., Biomaterials in bone surgery. (in Polish), (Silesian University of Technology, Gliwice, 1992)

12. A. Woźna, Current Problems of Biomechanics (in Polish), 5, 183 (2011)

13. A. Łukaszczyk, J. Augustyn-Pieniazek, Archives of metal and materials, 60 (2015)

14. ***Toxicological profile for nickel U.S. Dep. of health and human services Public Health Service Agency for Toxic Substances and Disease Registry, (2005)

15. R.D. Barrett, S.E. Bishara, Am. J. Orthod. Dentofacial Orthop. 103, 8-14 (1993)

16. Y Ocazaki, E Gotoh, Biomaterials 26, 11-21 (2005)

17. A. Banu, O. Radovici, M. Marcu, Roum. Biotechnol. Lett. 9, 1603-1608 (2004) 